\title{
THE LINK BETWEEN REGULARITY AND STRONG-PI-REGULARITY
}

\author{
PEDRO PATRÍCIO ${ }^{凶}$ and R. E. HARTWIG
}

(Received 23 December 2009; accepted 5 June 2010)

Communicated by B. J. Gardner

\begin{abstract}
It is shown that if all powers of a ring element $a$ are regular, then $a$ is strongly pi-regular exactly when a suitable word in the powers of $a$ and their inner inverses is a unit.
\end{abstract}

2000 Mathematics subject classification: primary 15A09; secondary 16A30.

Keywords and phrases: Drazin inverse, strongly pi-regular, Drazin index.

\section{Introduction}

An element $m$ in a ring $R$ is said to be regular if there exists $m^{-}$, referred to as an inner inverse, such that $\mathrm{mm}^{-} m=m$. The set of all inner inverses of $m$ is denoted by $m\{1\}$. We say that $m$ is strongly pi-regular if it has a Drazin inverse $m^{d}$ that satisfies $x m x=x$ and $m x=x m$, as well as $m^{k} x m=m^{k}$ for some $k$ [2]. The smallest such $k$ is called the index of $m$ and is denoted by $i(m)$. When $i(m) \leq 1$, we say that $m$ has a group inverse, and this is denoted by $m^{\#}$. In particular, $m$ is a unit if and only if $i(m)=0$. The index $i(m)$ can also be characterized as the smallest $k$ for which there exist $x$ and $y$ such that $a^{k+1} x=a^{k}=y a^{k+1}$. Given ring elements $x$ and $y$, we say they are orthogonal, and we write $x \perp y$, if $x y=y x=0$.

It is known that if $m$ is strongly pi-regular, then $m^{i(m)}$ is regular, and in fact belongs to a multiplicative group, which ensures that $\left(m^{i(m)}\right)^{\#}$ exists. We propose to solve the converse problem, namely, that of characterizing strong-pi-regularity in terms of the regularity of suitable powers of $m$ together with the existence of a word, in powers of $m$ and their inner inverses, that is a unit.

This research received financial support from the Research Centre of Mathematics of the University of Minho (CMAT) through the FCT Pluriannual Funding Program.

(C) 2010 Australian Mathematical Publishing Association Inc. 1446-7887/2010 \$16.00 


\section{The regular stack}

Suppose $m$ is an element in $R$, and assume that $m$ and all its powers are regular. For each power, we pick a fixed inner inverse. That is, we fix a list

$$
\left\{m^{-},\left(m^{2}\right)^{-}, \ldots,\left(m^{k}\right)^{-}, \ldots\right\} \text {. }
$$

We define the fixed idempotents $E_{k}=m^{k}\left(m^{k}\right)^{-}$, when $k=1,2, \ldots$, and we also set $e=E_{1}=m m^{-}$. It is easily seen that

$$
e m=m, \quad e E_{k}=E_{k}=E_{k}^{2}, \quad E_{k} m E_{k}=m E_{k}, \quad E_{k} E_{k+1}=E_{k+1} .
$$

We now consider the map $\phi: R \rightarrow R$ defined by $\phi(x)=m x e+1-e x e$, and construct the sequence of elements $m_{k}=\phi\left(E_{k}\right)=x_{k}+y_{k}$, where $x_{k}=m E_{k} e$ and $y_{k}=1-e E_{k} e$, when $k=1,2, \ldots$ Observe that $\phi(1)=\phi(e)$. We recall that $\phi(e)$ is a unit precisely when $m$ has a group inverse [7], and that $\phi(a)$ is a unit exactly when am has a group inverse [3]. In addition, we see that

$$
\begin{gathered}
x_{k} y_{k}=m E_{k} e-m E_{k} e E_{k} e=0, \\
y_{k} x_{k}=m E_{k} e-e E_{k} e m E_{k} e=m E_{k} e-E_{k} m E_{k} e=0,
\end{gathered}
$$

and therefore we have an orthogonal splitting $m_{k}=x_{k}+y_{k}$.

We now claim that the elements $m_{k}$ are in fact regular and may be generated recursively.

Lemma 2.1. If $m_{k}=\phi\left(m^{k}\left(m^{k}\right)^{-}\right)$, then there exists an inner inverse $m_{k-1}^{-}$such that

$$
m_{k}=m_{k-1}^{2} m_{k-1}^{-}+1-m_{k-1} m_{k-1}^{-},
$$

with $m_{0}=m$.

PROOF. If $i \geq 1$, then we have $m_{i}=x_{i}+y_{i}$, in which both components are regular. Indeed, $y_{i}=1-m^{i}\left(m^{i}\right)^{-} m m^{-}$and so $y_{i}$ is idempotent, and $x_{i}$ has an inner inverse, namely, $m^{i}\left(m^{i+1}\right)^{-} m m^{-}$; calling this $x_{i}^{-}$, we deduce that $x_{i} x_{i}^{-}=m^{i+1}\left(m^{i+1}\right)^{-} m m^{-}$ and $y_{i} x_{i}^{-}=0$ since

$$
e E_{i} e m^{i}=m m^{-} m^{i}\left(m^{i}\right)^{-} m m^{-} m^{i}=m^{i} .
$$

We can, therefore, take $m_{k-1}^{-}=x_{k-1}^{-}+y_{k-1}$, and this in turn gives

$$
\begin{aligned}
m_{k} & =m^{k+1}\left(m^{k}\right)^{-} m m^{-}+1-m^{k}\left(m^{k}\right)^{-} m m^{-} \\
& =x_{k-1} x_{k-1} x_{k-1}^{-}+y_{k-1}+1-x_{k-1} x_{k-1}^{-}-y_{k-1} \\
& =\left(x_{k-1}+y_{k-1}\right)\left(x_{k-1} x_{k-1}^{-}+y_{k-1}\right)+1-\left(x_{k-1} x_{k-1}^{-}+y_{k-1}\right) \\
& =m_{k-1}^{2} m_{k-1}^{-}+1-m_{k-1} m_{k-1}^{-},
\end{aligned}
$$

as desired.

Using this lemma, we can now express $m_{k}$ alternatively:

$$
m_{k}=m^{k+1}\left(m^{k}\right)^{-} m m^{-}+1-m^{k}\left(m^{k}\right)^{-} m m^{-} .
$$




\section{Index results}

Let us now use the above regular stack to obtain suitable index results. Suppose that $m$ is strongly pi-regular, and consider the associated sequences

$$
\begin{gathered}
u_{k}=m^{k+1}\left(m^{k}\right)^{-}+1-m^{k}\left(m^{k}\right)^{-}, \\
w_{k}=m^{-} m^{k+1}\left(m^{k}\right)^{-} m+1-m^{-} m^{k}\left(m^{k}\right)^{-} m, \\
v_{k}=\left(m^{k}\right)^{-} m^{k+1}+1-\left(m^{k}\right)^{-} m^{k} .
\end{gathered}
$$

We shall need the following fact.

Lemma 3.1 [1]. If $1+a b$ has a Drazin inverse, then $1+$ ba has a Drazin inverse and

$$
i(1+a b)=i(1+b a)
$$

PROOF. Suppose $1+a b$ has a Drazin inverse and its index $i(1+a b)$ is equal to $k$. Then

$$
(1+a b)^{k+1} x=(1+a b)^{k}=y(1+a b)^{k+1},
$$

for some $x$ and $y$ in $R$. This means that

$$
(1+b a)^{k+1}(1-b x a)=(1+b a)^{k}=(1-b y a)(1+b a)^{k+1},
$$

and thus $i(1+b a) \leq i(1+a b)$. By interchanging $a$ and $b$, we obtain the equality.

By applying this lemma we may conclude that $i\left(m_{k}\right)=i\left(u_{k}\right)=i\left(w_{k}\right)=i\left(v_{k}\right)$.

We now recall the following lemma.

LEMMA 3.2 [5]. If $m$ is strongly pi-regular, then

$$
i\left(m^{2} m^{-}+1-m m^{-}\right)=i(m)-1 .
$$

As a consequence, we may deduce that $i\left(m_{k}\right)=t$ if and only if $i\left(m_{k+1}\right)=t-1$.

We shall also need the following result, which can be deduced from the proof of [2, Theorem 4].

Lemma 3.3. If $a^{k+1} x=a^{k}=y a^{k+1}$, then we have $a^{d}=a^{k} x^{k+1}=y^{k+1} a^{k}$ and $a a^{d}=a^{k} x^{k}=y^{k} a^{k}$.

PROOF. Repeatedly premultiplying the first equality by $a$ and postmultiplying it by $x$ shows that $a^{k+r} x^{r}=a^{k}$ when $r=1,2, \ldots$, and in particular, if $r=k$, then $a^{2 k} x^{k}=a^{k}$. By symmetry, $a^{k}=y^{k} a^{2 k}$. The latter two equalities ensure that $a^{k}$ has a group inverse of the form

$$
\left(a^{k}\right)^{\#}=y^{k} a^{k} x^{k}=y^{k} a^{2 k} x^{2 k}=a^{k} x^{2 k}=y^{2 k} a^{k} .
$$

This implies that

$$
a^{d}=a^{k-1}\left(a^{k}\right)^{\#}=a^{k-1} a^{k} x^{2 k}=\left(a^{k+(k-1)} x^{k-1}\right) x^{k+1}=a^{k} x^{k+1},
$$

and by symmetry $a^{d}=y^{k+1} a^{k}$.

Finally, we also see that $a a^{d}=a^{k+1} x^{k+1}=\left(a^{k+1} x\right) x^{k}=a^{k} x^{k}$, and so $a a^{d}=y^{k} a^{k}$ by symmetry. 
Combining these results, we now may state the following theorem.

THEOREM 3.4. The following conditions are equivalent.

(a) $i(m)=s$.

(b) $s$ is the smallest integer such that $m^{s}+1-m^{s}\left(m^{s}\right)^{-}$is a unit.

(c) $s$ is the smallest integer such that $m^{2 s}\left(m^{s}\right)^{-}+1-m^{s}\left(m^{s}\right)^{-}$is a unit.

(d) $s$ is the smallest integer such that $m_{s}$ is a unit.

(e) $s$ is the smallest integer such that $u_{s}$ is a unit.

(f) $m_{\ell}$ is strongly pi-regular and $i\left(m_{\ell}\right)=s-\ell$, for one and hence all $\ell$ such that $0 \leq \ell \leq s$.

(g) $u_{\ell}$ is strongly pi-regular and $i\left(u_{\ell}\right)=s-\ell$, for one and hence all $\ell$ such that $0 \leq \ell \leq s$.

If the conditions are satisfied, then

$$
\begin{aligned}
m^{d} & =u_{s}^{-1} m^{s} v_{s}^{-s}=m^{s} v_{s}^{-s-1}=u_{s}^{-s} m^{s} v_{s}^{-1}=u_{s}^{-s-1} m^{s} \\
& =m^{s-1} u_{s}^{-(s+1)} m^{s+2} v_{s}^{-(s+1)} .
\end{aligned}
$$

PROOF. The equivalences between (a), (b) and (c) are known (see [6]). Since $i\left(m_{\ell}\right)=t$ if and only if $i\left(m_{\ell+1}\right)=t-1$, we may, by using this argument recursively, conclude that $i(m)=s$ if and only if $i\left(m_{\ell}\right)=s-\ell$.

The equivalence of (f) and (g), and that of (d) and (e), may be seen by applying Lemma 3.1, setting $b=m m^{-}$and first $a=m^{\ell+1}\left(m^{\ell}\right)^{-}-m^{\ell}\left(m^{\ell}\right)^{-}$and then $a=m^{s+1}\left(m^{s}\right)^{-}-m^{s}\left(m^{s}\right)^{-}$. It is obvious that (f) implies (d) and that (g) implies (e).

Finally, we now prove that (e) implies (a). As $u_{s}$ is a unit and $u_{s} m^{s}=m^{s+1}$, we have $m^{s}=u_{s}^{-1} m^{s+1}$. Likewise, $v_{s}=\left(m^{s}\right)^{-} m^{s+1}+1-\left(m^{s}\right)^{-} m^{s}$, so $u_{s}$ being a unit implies that $v_{s}$ is a unit, and this in turns yields $m^{s}=m^{s+1} v_{s}^{-1}$. Therefore, $m^{s} \in m^{s+1} R \cap R m^{s+1}$ and $m^{d}=m^{s-1} u_{s}^{-(s+1)} m^{s+2} v_{s}^{-(s+1)}$.

We may in fact compute the Drazin inverses of the three associated sequences $\left\{u_{k}\right\},\left\{v_{k}\right\}$ and $\left\{w_{k}\right\}$. It suffices to compute the former.

THEOREM 3.5. If $i(m)=s$ and $0 \leq \ell \leq s$, then

$$
u_{\ell}^{d}=m^{d} m^{\ell}\left(m^{\ell}\right)^{-}+1-m^{\ell}\left(m^{\ell}\right)^{-} .
$$

Proof. Set $X=m^{\ell}$ and $A=m\left(m^{\ell}\right)^{-}$, so that $u_{\ell}=X A+\left(1-E_{\ell}\right)$. From the last theorem, we recall that $i\left(u_{\ell}\right)=i(m)-\ell$. Now observe that $u_{\ell}$ is a sum of two orthogonal elements, and since $u_{\ell}$ is strongly pi-regular, so are each of the two orthogonal summands. In particular, $m^{\ell+1}\left(m^{\ell}\right)^{-}$is strongly pi-regular and we obtain the expression

$$
\left(u_{\ell}\right)^{d}=\left(m E_{\ell}\right)^{d}+1-E_{\ell}=(X A)^{d}+1-E_{\ell},
$$

where $E_{\ell}=m^{\ell}\left(m^{\ell}\right)^{-}$. 
Next, we turn our attention to the computation of $(X A)^{d}=\left(m E_{\ell}\right)^{d}$. We claim that $(X A)^{k+1} y=(X A)^{k}$, where $y=m^{d} m^{\ell}\left(m^{\ell}\right)^{-}$. Indeed, it follows by induction that $(X A)^{i}=m^{i+\ell}\left(m^{\ell}\right)^{-}$, and hence

$$
\begin{aligned}
(X A)^{k+1} y & =m^{k+\ell+1}\left(m^{\ell}\right)^{-} m^{\ell} m^{d}\left(m^{\ell}\right)^{-}=m^{\ell} m^{k+1} m^{d}\left(m^{\ell}\right)^{-} \\
& =m^{k+\ell}\left(m^{\ell}\right)^{-}=(X A)^{k} .
\end{aligned}
$$

We now apply Lemma 3.3 to obtain $(X A)^{d}=(X A)^{k} y^{k+1}$.

Again, by induction, $y^{i}=\left(m^{d}\right)^{i} m^{\ell}\left(m^{\ell}\right)^{-}$, whence $y^{k+1}=\left(m^{d}\right)^{k+1} m^{\ell}\left(m^{\ell}\right)^{-}$, and this gives

$$
\begin{aligned}
(X A)^{d} & =(X A)^{k} y^{k+1}=m^{\ell+k}\left(m^{\ell}\right)^{-}\left(m^{d}\right)^{k+1} m^{\ell}\left(m^{\ell}\right)^{-} \\
& =m^{\ell+k}\left(m^{\ell}\right)^{-} m^{\ell}\left(m^{d}\right)^{k+1}\left(m^{\ell}\right)^{-}=m^{\ell} m^{k}\left(m^{d}\right)^{k+1}\left(m^{\ell}\right)^{-} \\
& =m^{d} m^{\ell}\left(m^{\ell}\right)^{-}
\end{aligned}
$$

and

$$
(X A)^{d} X A=\left(m E_{\ell}\right)^{d} m E_{\ell}=m^{d} m^{\ell}\left(m^{\ell}\right)^{-} m^{\ell+1}\left(m^{\ell}\right)^{-}=m^{d} m^{\ell+1}\left(m^{\ell}\right)^{-} .
$$

Finally, substituting the expression for $(X A)^{d}$ in (3.1), we arrive at

$$
\left(u_{\ell}\right)^{d}=m^{d} E_{\ell}+1-E_{\ell}=m^{d} m^{\ell}\left(m^{\ell}\right)^{-}+1-m^{\ell}\left(m^{\ell}\right)^{-},
$$

which is the desired expression.

We close with some pertinent remarks.

\section{Remarks}

(a) If $m_{k}$ is a unit for one choice of $\left(m^{k}\right)^{-}$, then it is a unit for all such choices. Indeed, the fact that $m_{k}$ is a unit implies that $i(m)=s$, which implies, from the proof of Theorem 3.4, that $m_{s}=m^{s+1}\left(m^{s}\right)=m m^{-}+1-m^{s}\left(m^{s}\right)^{=} m m^{-}$is also a unit.

(b) If $u_{s}$ is a unit for one choice of $\left(m^{s}\right)^{-}$, then it is a unit for all such choices.

(c) In a ring, $a^{2}$ may be regular without $a$ being regular. For example, take $a=4$ in $\mathbb{Z}_{8}$.

(d) In a ring, $a$ may be regular without $a^{2}$ being regular. Indeed, in $\mathbb{Z}_{4}$, consider

$$
A=\left[\begin{array}{ll}
0 & 0 \\
1 & 2
\end{array}\right] \quad \text { and } \quad B=\left[\begin{array}{ll}
0 & 1 \\
0 & 0
\end{array}\right]
$$

Then $A$ has an inner inverse, namely $B$, but $A^{2}$ has no inner inverse, since $A^{2}=2 A$, and so $(2 A) X(2 A)=0 \neq 2 A$.

\section{Acknowledgements}

The authors wish to thank the referee and the editor for their valuable corrections and comments. 


\section{References}

[1] N. Castro-González, C. Mendes Araújo and P. Patrício, 'A note on generalized inverses of a sum in rings', Bull. Aust. Math. Soc. 82 (2010), 156-164.

[2] M. P. Drazin, 'Pseudo-inverses in associative rings and semigroups', Amer. Math. Monthly 65 (1958), 506-514.

[3] F. J. Hall and R. E. Hartwig, 'Algebraic properties of governing matrices used in Cesàro-Neumann iterations', Rev. Roumaine Math. Pures Appl. 26(7) (1981), 959-978.

[4] R. E. Hartwig, 'More on the Souriau-Frame algorithm and the Drazin inverse', SIAM J. Appl. Math. 31(1) (1976), 42-46.

[5] P. Patrício and A. Veloso da Costa, 'On the Drazin index of regular elements', Cent. Eur. J. Math. 7(2) (2009), 200-205.

[6] R. Puystjens and M. C. Gouveia, 'Drazin invertibility for matrices over an arbitrary ring', Linear Algebra Appl. 385 (2004), 105-116.

[7] R. Puystjens and R. E. Hartwig, 'The group inverse of a companion matrix', Linear Multilinear Algebra 43(1-3) (1997), 137-150.

PEDRO PATRÍCIO, Departamento de Matemática e Aplicações,

Universidade do Minho, 4710-057 Braga, Portugal

e-mail: pedro@math.uminho.pt

R. E. HARTWIG, Department of Mathematics, N.C.S.U., Raleigh,

NC 27695-8205, USA

e-mail: hartwig@unity.ncsu.edu 